\title{
The Forgotten Remit of Astrobiology
}

\section{Chandra Wickramasinghe *}

Buckingham Centre for Astrobiology, University of Buckingham, Buckingham, UK

\begin{abstract}
Astrobiology as it is currently pursued generally ignores the possibility that life might be a cosmic phenomenon, and that fully-fledged microbial life - bacteria and viruses - are cosmically omnipresent. The exploration of this possibility, which is opposed to the conventional view of an origin of life occurring independently on individual planets like Earth, is urgently needed in the light of new data.
\end{abstract}

Keywords: Astrobiology; Panspermia; Life as a cosmic phenomenon; Fred hoyle

\section{Introduction}

From the mid-1970's onwards the present author and the late Sir Fred Hoyle argued that spectroscopic evidence existed for the widespread occurrence of organic molecules and biomolecules throughout the interstellar medium of the galaxy [1-5]. Although our claims were refuted at the time it is now conceded without dissent that such molecules exist everywhere [6,7]. But how are these molecules formed? And what, if any, is their connection with life. The consensus, is that these molecules form inorganically and represent the precursors of biology. When prebiotic molecules are delivered to planets like the Earth via comets and meteorites [7] an origin of life takes place in the manner originally proposed by Oparin and Haldane [8].

Despite several decades of intense effort in the laboratory the Oparin-Haldane process - the primordial soup theory - has not been

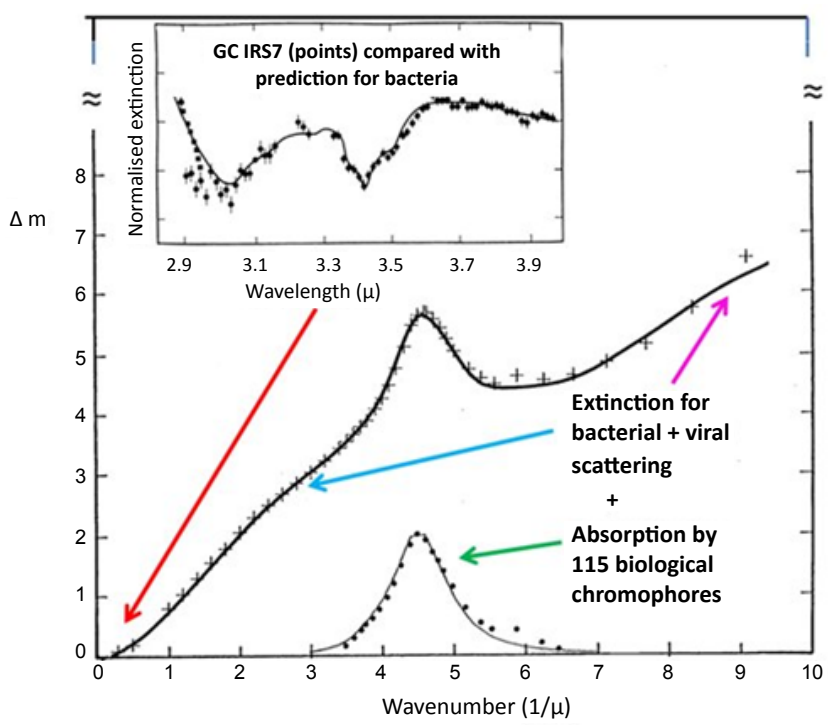

Figure 1: Interstellar extinction data - dimming of starlight due to absorption and scattering by cosmic dust - (points) compared with model involving desiccated bacteria, viral-sized organic particles and aromatic molecules resulting from the degradation of biomaterial. The curves represent the predicted behaviour of the biological dust models. The blue and purple arrows point to segments of the extinction curve in the visual and ultraviolet wavebands; the red arrow points to the inset which is an enlargement of the extinction curve near the wavenumber 0.3 inverse microns; the green arrow points to the hump in the extinction curve which is fitted to biological aromatic molecules (11,14 for details). convincingly proved [9]. The alternative possibility is that fully-fledged microbial life exists on a galactic scale and that the astronomically observed molecules are mostly the detritus of biology. This latter possibility is not considered by mainstream astrobiology because it is seen to fly in the face of orthodoxy.

The earliest astronomical evidence supporting this stronger and more daring possibility was the infrared spectrum of the galactic centre infrared source GC-IRS7, as well as the extinction curve of starlight which together pointed to biology on a cosmic scale $[10,11]$. The implication of the model fits of the type shown in Figure 1 is that some $20 \%$ or more of the mass of carbon in interstellar space is tied up in the form of particles that are spectroscopically indistinguishable from freeze-dried bacteria, viruses and their degradation products [12-14].

It was such astronomical evidence combined with the lack of progress towards understanding the origin of life at the molecular level that first prompted the exploration of life as a cosmic phenomenon. The improbabilities associated with the origin of life are then safely tucked away in an early stage in the evolution of the Big-Bang Universe [13] or, life was ever present in a universe with an open timescale [14]. In a lecture entitled "The relation of biology to astronomy" delivered at an out-of-town meeting of the Royal Astronomical Society in Cardiff on 15 ${ }^{\text {th }}$ April 1980, Fred Hoyle concluded thus: "Microbiology may be said to have had its beginnings in the nineteen-forties. A new world of the most astonishing complexity began then to be revealed. In retrospect I find it remarkable that microbiologists did not at once recognize that the world into which they had penetrated had of necessity to be of cosmic order. 1 suspect that the cosmic quality of microbiology will seem as obvious to future generations as the Sun being the center of the solar system seems obvious to the present generation".

This lecture was essentially an unofficial launch of the new subject of astrobiology some years in advance of its formal recognition as a new scientific discipline. Modern explorations of astrobiology have been mainly concerned with studying the extreme survival properties of bacteria that make them fit for space travel, and also with locating suitable astronomical habitats for life within the solar system and

*Corresponding author: Chandra Wickramasinghe, Buckingham Centre for Astrobiology, University of Buckingham, Buckingham, UK, Tel: 44-777-38-9243; E-mail: ncwick@gmail.com

Received June 18, 2014; Accepted June 20, 2014; Published June 22, 2014

Citation: Wickramasinghe C (2014) The Forgotten Remit of Astrobiology. Astrobiol Outreach 2: e105. doi: 10.4172/2332-2519.1000e105

Copyright: (C) 2014 Wickramasinghe C. This is an open-access article distributed under the terms of the Creative Commons Attribution License, which permits unrestricted use, distribution, and reproduction in any medium, provided the original author and source are credited. 

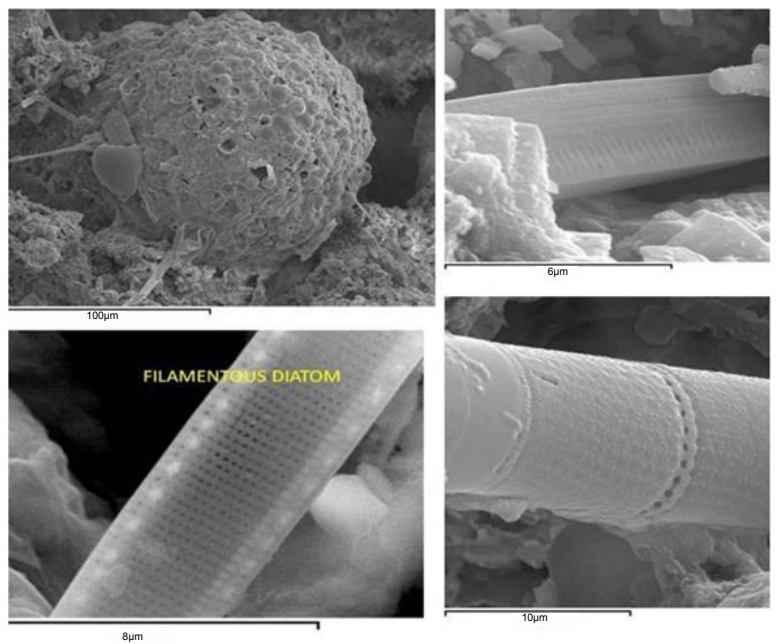

Figure 2: Fossilised acritarch (extinct microorganism) at top left and diatom fossils in the 2012 Sri Lankan (Polonnaruwa) meteorite.

beyond. The implicit premise, which is unproven, is that given the appropriate physical and chemical condition life must originate inevitably in the manner of the Oparin-Haldane model. The stronger possibility that fully-fledged microbial life is omnipresent, as implied in the above quotation and in Figure 1, is rarely discussed let alone explored.

The original evidence from astronomy for the theory of cosmic life [12-14] has subsequently been backed up by studies of meteorites and cometary dust. Studies of meteorites by Pflug in the 1980's that revealed the unequivocal presence of fossilized bacteria and viruses [15] have been supported by a recent study of a meteorite that fell in Sri Lanka in December 2012. Examination of this meteorite by Jamie Wallis and colleagues leaves little room to doubt that fossilized microbiological entities including the highly characteristic morphologies of diatoms are present [16]. Some of these structures are reproduced in Figure 2. Critics who have argued that the stones in question could not be meteorites (presumably because they contain life) would have to explain many facts that militate against their terrestrial origin. Witness reports of a fireball sighting that preceded the fall, non-terrestrial ratios of the stable oxygen isotopes, high abundances of the element iridium are just a few. Another recent piece of evidence of a similar kind was obtained by Milton Wainwright and his colleagues [17,18] showing biological entities in cometary meteoroids collected from $27 \mathrm{~km}$ in the stratosphere. All this shows clearly that it may be unwise to continue ignoring the concept of life being a cosmic phenomenon. Extending the remit of astrobiology to include an examination of this possibility is certainly long overdue.

\section{References}

1. Wickramasinghe NC (1974) Formaldehyde polymers in interstellar space. Nature 252: 462-463.

2. Hoyle F, Wickramasinghe NC (1976) Primitive grain clumps and organic compounds in carbonaceous chondrites. Nature 264: 45-46.

3. Hoyle F, Wickramasinghe NC (1977) Polysaccharides and the infrared spectra of galactic sources, Nature 268: 610-612.

4. Hoyle F, Wickramasinghe NC (1977) Identification of the 2200A interstellar absorption feature, Nature 270: 323

5. Hoyle F, Wickramasinghe NC (1986) The case for life as a cosmic phenomenon Nature 322: 509-511.

6. Kwok S (2009) Organic matter in space: from stardust to the solar system Astrophys Space Sci. 319: 5-21.

7. Philippe SK, Zelimir G, Régis DG, Agnes F, Basem K, et al. (2010) High molecular weight diversity in the extraterrestrial organic matter in the Murchison Meteorite, revealed 40 years after its fall. PNAS 107: 2763-2768.

8. Oparin AI (1953) The Origin of Life (transl. S. Marguli), Dover, NY.

9. Deamer D (2011) First Life. California University Press, Berkley, USA.

10. Hoyle F, Wickramasinghe NC, Al-Mufti S (1984) The spectroscopic identification of interstellar grains. Astrophys. Sp.Sci. 98: 343-352.

11. Wickramasinghe NC (2010) The astrobiological case for our cosmic ancestry Int.J.Astrobiol 9: 119-129.

12. Hoyle F, Wickramasinghe NC (1982) Proofs that Life is Cosmic.

13. Gibson CH, Schild RE, Wickramasinghe NC (2011) The origin of life from primordial planets. Int. J. Astrobiol. 10: 83-98.

14. Hoyle F and Wickramasinghe NC (2000) Astronomical Origins of Life: Steps towards Panspermia. Kluwer Academic Press, Dordrecht, Netherlands.

15. Pflug HD (1984) In: N.C. Wickramasinghe, ed. Fundamental Studies and the Future of Science, Cardiff: Univ. College Cardiff Press, USA.

16. Jamie W, Nori M, Richard BH, Andrew O, Daryl HW, et al. (2013) The Polonnaruwa meteorite: oxygen isotope, crystalline and biological composition. Journal of Cosmology.

17. Wainwright M, Rose CE, Baker AJ, Briston KJ, Wickramasinghe NC (2013) Isolation of a diatom frustules from the lower stratosphere $(22-27 \mathrm{~km})$. Journal of Cosmology 22: 10183-10188.

18. Wainwright M, Rose CE, Baker AJ, Wickramasinghe NC (2013) Isolaton of biological entities from the stratosphere $(22-27 \mathrm{~km})$. Journal of Cosmology 22 : 10189-10197. 